\title{
EXPERIMENTAL EVIDENCE OF AN ELECTRON TEMPERATURE ENHANCEMENT IN THE WAKE OF AN IONOSPHERIC SATELLITE
}

\author{
URI SAMIR \\ Space Physics Research Laboratory, University of Michigan, Ann Arbor, Michigan 48105, U.S.A. \\ and \\ Department of Environmental Sciences, Tel-Aviv University, Ramat-Aviv, Israel \\ and \\ G. L. WRENN* \\ The University of Texas at Dallas, Box 30365, Dallas, Texas 75230, U.SA.
}

(Received in final form 30 December 1971)

\begin{abstract}
A study of electron temperature $\left(T_{e}\right)$ measurements made by a Langmuir probe mounted on the skin of the Explorer 31 satellite indicates that electron temperature in the very near wake exceeds that of the ambient electron gas. Potential causes of such an enhancement are mentioned but the possibility that the wake 'temperatures' refiect the existence of nonMaxwellian energy distributions cannot be discounted. The results are compared with observations from the Gemini/Agena wake experiment and are found to be in reasonable agreement.
\end{abstract}

\section{INTRODUCTION}

Studies of the plasma wakes produced by the motion of spacecraft through the ionosphere have been reviewed by Gurevich et al. (1969). At present there are serious inadequacies in the available theories but relatively few experimental data which can be employed to obtain a better description of such wakes. It is no accident that satellite experiments are designed to avoid the problems encountered in the wake.

In this paper measurements of electron temperature $\left(T_{e}\right)$ from the planar Langmuir probe on board the Explorer 31 satellite (Wrenn, 1969) have been used to investigate the angular distribution, $T_{e}=f(\theta)$, where $\theta$ is the angle between the probe normal and the velocity vector (angle of attack).

The satellite is basically an octagonal parallelepiped of height $62 \mathrm{~cm}$, the distance between opposite faces being $87 \mathrm{~cm}$. The electron energy probe is located in the centre of one face $13.5 \mathrm{~cm}$ above the base plane, see Samir and Wrenn (1969). The cart-wheel mode of spin stabilisation of the satellite produced a continual sampling of the wake region; the nominal spin period was $20 \mathrm{sec}$ whilst the sweep period of the electron probe was $7.5 \mathrm{sec}$.

The experimental technique and method of analysis have been described elsewhere (Boyd, 1968; Wrenn, 1969; Willmore, 1970) but there are some limitations which must be considered when dealing with wake currents. The instrumentation electronically determines the slope of the semi-log collector current-voltage characteristic; normally this slope $\left(\alpha 1 / T_{e}\right)$ is constant over a range of retarding voltages close to plasma potential indicating the Maxwellian nature of the electron energy distribution: departures from Maxwellian are thus detected by variations in the slope (modulation depth) output. In the wake situation currents are drastically reduced and the sensitivity limit of the amplifier can be encountered; this means that fewer data points are available for $T_{\theta}$ determination resulting in a limited sampling of the velocity distribution and larger error bars; it is no longer possible to adequately check for deviations from Maxwellian. It seems probable that such deviations

* On leave from Mullard Space Science Laboratory, University College London. 


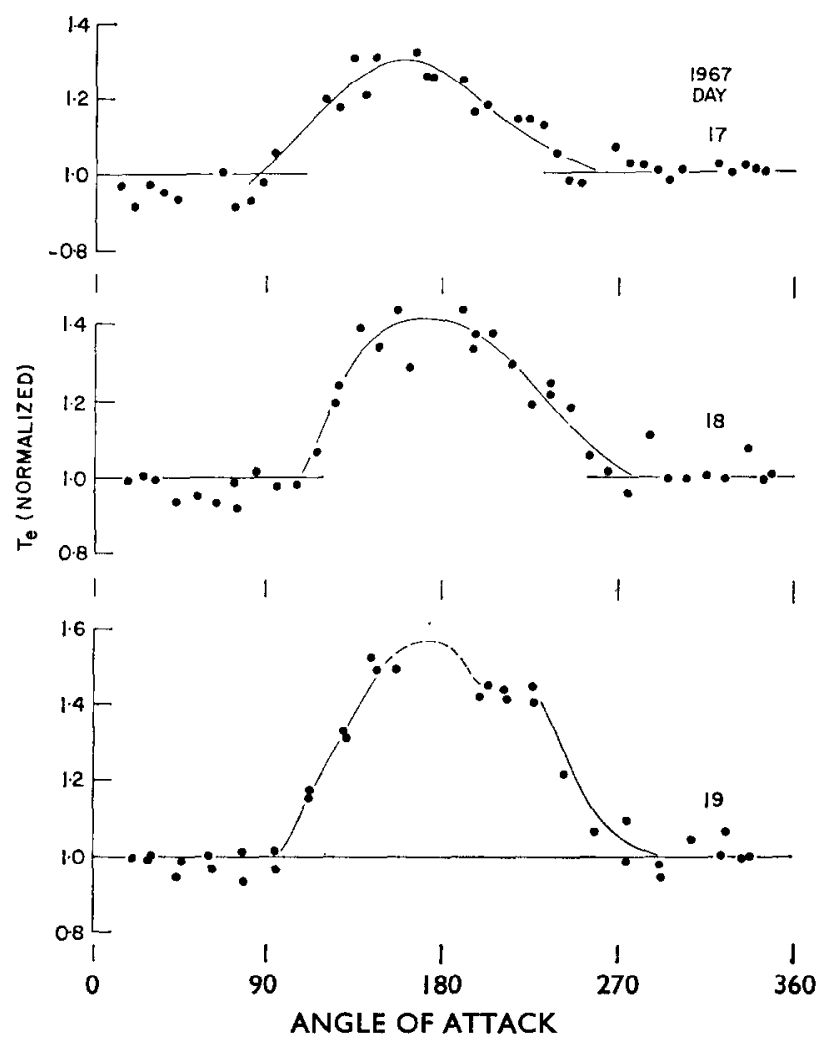

FIg. 1. VARIATION OF NORMALISED ELECTRON TEMPERATURE WITH ANGLE OF ATTACK.

(Altitude $=800-1000 \mathrm{~km}$; Dominant ion $=0^{+}$; Satellite velocity $=7 \cdot 9 \mathrm{~km} / \mathrm{sec}$ ).

do occur in the wake, in which case the temperatures described below do not completely characterise the electron populations. Banks (1968) described non-Maxwellian hydrogen ion velocity distributions and showed that a 'temperature' parameter could still be usefully employed. Here 'temperature' is taken as inversely proportional to the maximum modulation depth of the probe signal current; it is believed that this is an appropriate measure of the electron energy distribution provided that no vast departure from Maxwellian exists. $T_{e}$ is used without quotation marks in the succeeding sections in order to avoid pedanticism but the possible qualification should not be forgotten.

\section{OBSERVATIONS AND ANALYSIS}

With a satellite spin period of $20 \mathrm{sec}$, only three angle of attacks are sampled each spin cycle and the fast rate of change of $\theta$ makes it difficult to deduce $T_{e}=f(\theta)$ accurately. Fortunately the spin rate was decreased on occasions but in order to obtain sufficient points to define the function it is necessary to combine data from a number of spin cycles. Generally the ambient temperature, i.e. $T_{e}$ (front), varies through a pass and all data must be normalised to take account of this. Figure 1 shows three examples of such normalised $T_{e}$ 's plotted against $\theta$. These results are from January 1967 when the satellite stabilisation was close to nominal although the spin period was approximately $52 \mathrm{sec}$. It is clearly seen that $T_{e}$ (wake) is greater than $T_{e}$ (front) and that the enhancement is as much as 50 per cent in the $\theta=180^{\circ}$ position. 

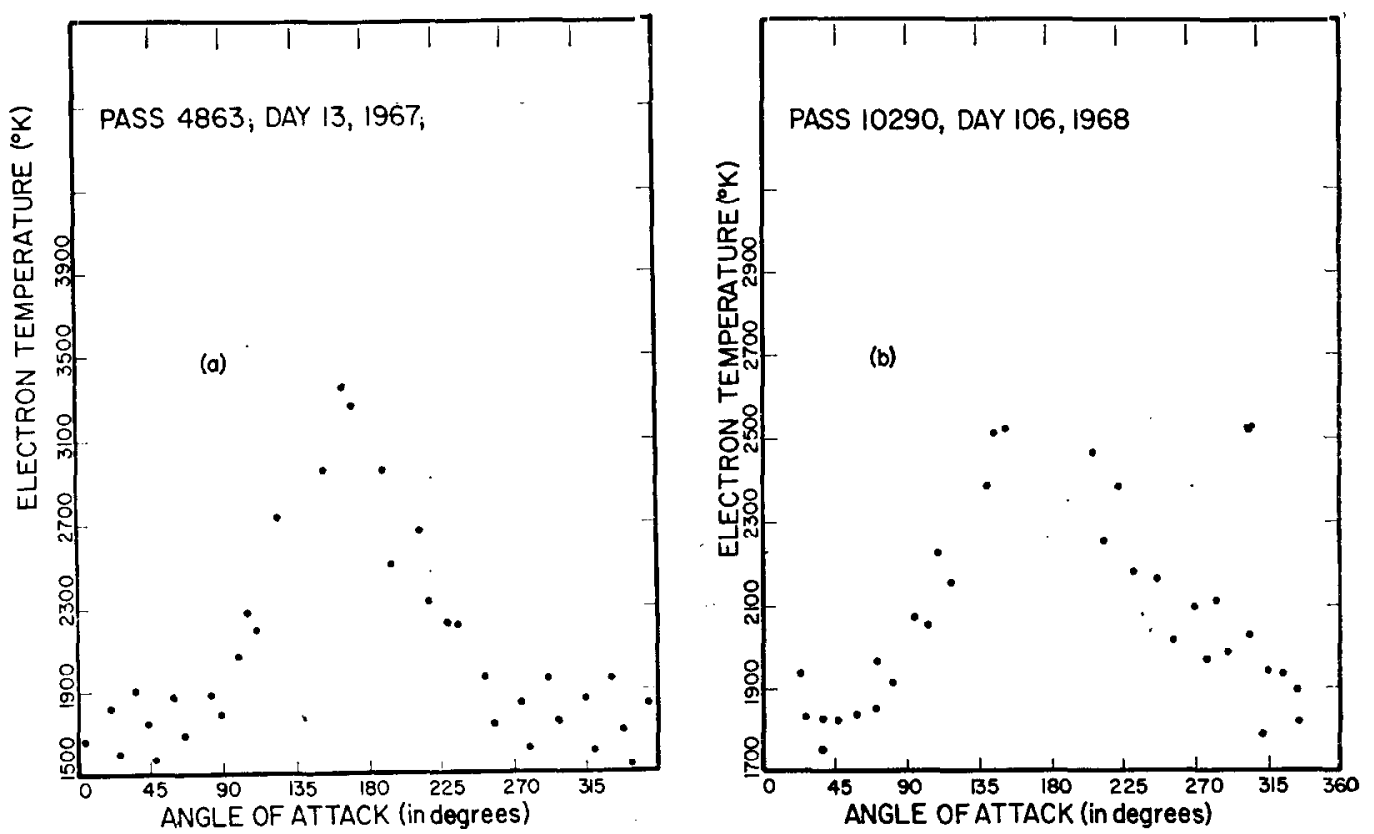

Fig. 2. VARIATION OF ELECTRON TEMPerature WTTH ANGLF, OF ATtACK.

$\begin{array}{ll}\text { (a) } \mathrm{O}^{+} \text {Plasma } & \text { (b) } \mathrm{H}^{+} \text {Plasma }\end{array}$

Figure 2 shows examples of $T_{e}=f(\theta)$ taken (a) close to $1000 \mathrm{~km}$ where $\mathrm{O}^{+}$is dominant and the satellite velocity, $V_{s}$, equals $7.7 \mathrm{~km} / \mathrm{sec}$ and (b) at $2800 \mathrm{~km}$ where $\mathrm{H}^{+}$is dominant and $V_{s}=6.1 \mathrm{~km} / \mathrm{sec}$. Although the variation in $T_{e}$ through these passes was not sufficient to necessitate the normalisation procedure it does introduce some scatter on the points. A number of plasma parameters which determine wake formation are listed in Table 1 and serve to demonstrate that the $T_{e}$ (wake) enhancements are evident through a considerable range of these values. Example (a) presents the maximum enhancement ever seen in the data analysed.

When the satellite spin period is nearer $20 \mathrm{sec}$ it is still possible to show the same effect by plotting $T_{e}$ through a pass for specific angle of attack intervals, as in Fig. 3. Temperatures appropriate to $\theta=180 \pm 15^{\circ}$ or $\theta=180 \pm 30^{\circ}$ are consistently higher than those for $\theta=0 \pm 30^{\circ}$ or $\theta=0 \pm 60^{\circ}$. It has been shown (Samir et al., 1971) that the ratio $T_{e}$ (wake) $T_{e}$ (front) averaged over a large number of locations and times, significantly exceeds $1 \cdot 0$ for altitudes below $2000 \mathrm{~km}$. At higher altitudes the enhancement is less obvious.

The same electron energy probe experiment was flown on the Ariel I satellite but was boom mounted approximately $5 R_{0}\left(R_{0}=\right.$ satellite radius) from the spacecraft centre. Henderson and Samir (1967) reported that they could not detect any deviations from the ambient electron temperature in the wake indicating that the $T_{e}$ (wake) enhancement does not extend very far downstream.

The determination of $T_{e}$ as a function of $\theta$ is sometimes complicated by a dependence on the angle, $\alpha$, between the probe normal and the magnetic field direction. Figure 4 illustrates the situation with two plots of normalised $T_{\varepsilon}$ against $\alpha$; the uncertainty limits of the data are shown and absolute $T_{e}$ scales are also indicated. Here the magnetic field vector is perpendicular to the spin axis of the satellite and $T_{\varepsilon}$ depressions are observed close to $\alpha=270^{\circ}$. The wake enhancements are also clearly visible and in fact they mask 


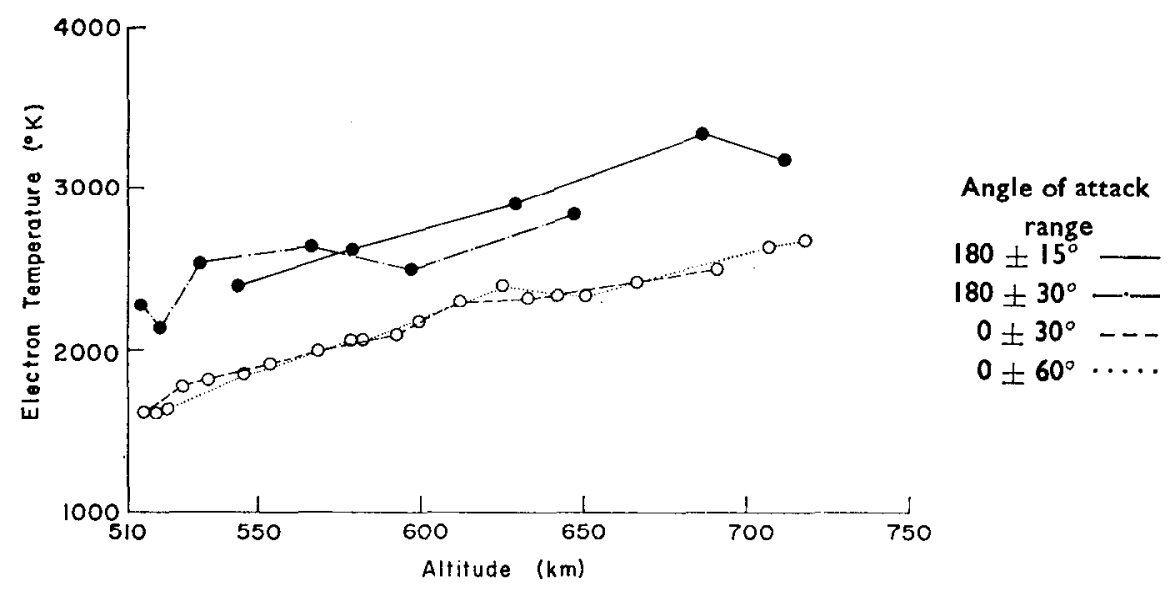

Fig. 3. Electron temperature versus altitude.

(Dominant ion $=0^{+}$; Satellite velocity $=8 \cdot 1 \mathrm{~km} / \mathrm{sec}$ ).

further depressions at $\alpha=30^{\circ}$. Bowling and Raitt (1969) have used a two probe system on the ESRO 1 satellite in which the planar probes were aligned parallel and normal to the magnetic vector. They confirm that $T_{e}$ (perpendicular) can be less than $T_{e}$ (parallel).

\section{DISCUSSION}

The $T_{e}$ (wake) measurements at the Explorer 31 surface can be compared with Medved's (1969) results from the Gemini/Agena wake experiment for the near wake zone. In manoeuyres where the Gemini capsule undocked from the Agena target vehicle, retarding potential analysers on the latter were used to measure the parameters of the wake created by the Gemini. Medved found $T_{e}$ (wake) $=2700^{\circ} \mathrm{K}$ greater than $T_{e}$ (ambient) $=1000^{\circ} \mathrm{K}$ in one case and $T_{e}$ (wake) $=1650^{\circ} \mathrm{K}>T_{e}$ (ambient) $=886^{\circ} \mathrm{K}$ in another. In view of the different wake dimension and geometry $\left(R_{0} \sim 80 \mathrm{~cm}\right)$ these enhancements are not incompatible with the Explorer 31 results. We know of no other published in situ observations for $T_{e}=f(\theta)$.

It has not been possible to account for the wake increases of electron temperature in terms of instrumental effects; the fact that these increases have been observed by two significantly different techniques is also pertinent.

Since no $T_{e}$ (wake) enhancement was found at $r=5 R_{0}$ (Henderson and Samir, 1967) it may be possible to conclude that the phenomenon is restricted to the near wake zone. This is supported by the results of Troy et al. (1970) and by the work of Gurevich et al. (1969) who used a 'neutral approximation' theory with $\left(M_{i} V_{s}^{2} / 2 k T_{e}\right)^{1 / 2}=3.75$ to satisfactorily explain that $r=5 R_{0}$ data.

The nature and cause of the 'temperature' enhancement is not yet clear. Behind the

Table 1. Relevant plasma parameters appropriate to Fig. 2

\begin{tabular}{ccccccc}
\hline & $\begin{array}{c}T_{e} \text { (front) } \\
\left({ }^{(} \mathrm{K}\right)\end{array}$ & $\begin{array}{c}N_{e} \text { (front) } \\
\left(\mathrm{cm}^{-3}\right)\end{array}$ & $\begin{array}{c}M_{i} \\
\text { (a.m.u.) }\end{array}$ & $\frac{e \phi}{k T_{e}}$ & {$\left[\frac{M_{i} V_{\mathrm{a}}^{2}}{2 k T_{e}}\right]^{1 / 2}$} & $\frac{R_{0}}{\lambda_{D}}$ \\
\hline a & 1740 & $1.2 \times 10^{4}$ & 12 & -8.4 & 5 & 15 \\
b & 1950 & $1 \cdot 1 \times 10^{3}$ & 1 & $-7 \cdot 2$ & $1 \cdot 1$ & $4 \cdot 3$ \\
\hline
\end{tabular}

$N_{a}$ (front) $=$ ambient electron density.

$M_{i}=$ mean ionic mass.

$\phi=$ satellite potential.

$R_{0}=$ satellite radius $=40 \mathrm{~cm}$.

$\lambda_{D}=$ Debye length. 


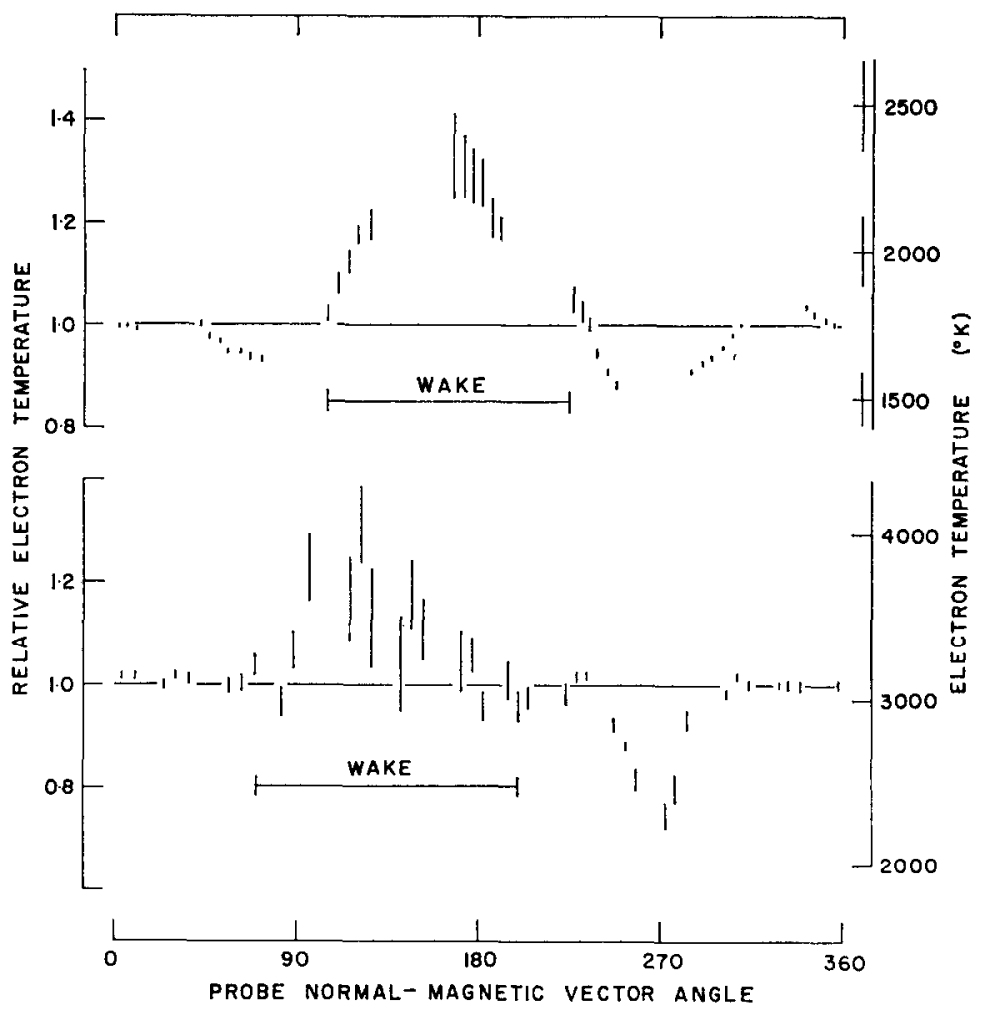

Fig. 4. Normalised electron temperature as a fUNCTION OF PROBE ORIENTATION WITH RESPECT TO THE MAGNETIC FIELD.

Wake regions are included.

satellite there exists a negative potential well (Liu, 1969) producing a potential gradient between itself and the more positive spacecraft. It is possible that in this well, wave-particle interactions take place and apply an energy filtering mechanism to the particles (electrons) that leave it. The resulting population close to the spacecraft can then have an effective temperature higher than that of the ambient thermal electrons. Alternatively, one might infer the existence of heating mechanisms in the wake region due to stream interactions and/ or instabilities correlated with plasma oscillations in the near wake (Samir et al., 1971).

These possibilities remain as yet speculative in nature until more theoretical and experimental work can be done; in particular, a measurement of the complete energy distribution of the wake population would clarify the situation and establish the true significance of the 'temperature' determinations.

Acknowledgements-We wish to thank Prof. R. L. F. Boyd and colleagues at the Mullard Space Science Laboratory, University College, London; in particular Prof. A. P. Willmore and Mr. C. L. Henderson for their interest and valuable communications. Thanks are due also to Mr. G. R. Carignan, Director of the Space Physics Research Laboratory at the University of Michigan, for useful discussions and for his interest and encouragement.

The work was supported in part by NASA under Grant NGR-23-005-320.

\section{REFERENCES}

Banks, P. M. (1968). Planet. Space Sci. 16, 759.

Bowling, T. S. and RAITT, W. J. ESRO/ELDO Bulln. ESRO-1 Supp. 9 April 1969.

BoYd, R. L. F. (1968). Plasma Diagnostics (Ed. Lochte-Holtgreven), p. 732. North-Holland, Amsterdam. Gurevich, A. V., Pitaeyski, L. P. and Smithova, V. V. (1969). Space Sci. Rev. 9(6) 805. 
Henderson, C. L. and Samir, U. (1967). Planet. Space Sci. 15, 1499.

LIU, V. C. (1969). Space Sci. Rev., 9, 423.

Medved, D. B. (1969). Rarefied Gas Dynamics, Sixth Int. Symp. Proc., p. 1525.

Samir, U., Henderson, C. L. and WrenN, G. L. Rarefied Gas Dynamics, 7th Int. Conf., Pisa, Italy. (Proc. to appear in 1971).

SAmir, U. and WrenN, G. L. (1969). Planet. Space Sci. 17, 693.

Troy, B. JR., MedVEd, D. B. and SAmir, U. (1970). J. astronaut. Sci. 18(3), 173.

Willmore, A. P. (1970). Space Sci. Rev., 11(5), 607.

WRENN, G. L. (1969). Proceedings of the IEEE 57(6), 1072. 